\title{
Preparation of TEG material based on conducting polymer PEDOT: PSS through treatment by nitric acid
}

\author{
S. M. Said ${ }^{1}$, S. M. Rahman ${ }^{1}$, B. D. Long ${ }^{2}$ \& M. F. M. Sabri ${ }^{2}$ \\ ${ }^{1}$ Department of Electrical Engineering, University of Malaya, Malaysia \\ ${ }^{2}$ Department of Mechanical Engineering, University of Malaya, Malaysia
}

\begin{abstract}
Sustainable energy sources are gaining popularity due to the ever increasing energy demand along with escalating costs and the environmental issues associated with the conventional sources of energy. Among the technologies available today, TEGs (Thermoelectric Generators) are intensively investigated due to their capability of harnessing electricity from waste heat, such as power plants, industries, automobiles and even human bodies. Our work focuses on the development of high efficiency organic thermoelectric materials to harness energy from ambient sources such as solar and domestic heat. In this present work, poly (3, 4-ethylenedioxythiophene)/(poly)styrenesulfonate (PEDOT: PSS) was post treated using nitric acid in order to increase its electrical conductivity, hence the efficiency. A maximum electrical conductivity of $197.51 \mathrm{~S} / \mathrm{cm}$ was achieved which was approximately 115 times than that of the untreated material. Improvement of the electrical conductivity after post-treatment is due to the depletion of the non-conducting PSS after the acid treatment. Hence, it is concluded that it is an easy method to achieve comparatively good thermoelectric properties which can find its uses in flexible thermoelectric applications.

Keywords: TEG, Seebeck effect, organic thermoelectric material, post treatment.
\end{abstract}

\section{Introduction}

Electricity is the prime mover of this modern world. The demand for electricity is increasing very fast each year. The 'Global Energy Statistical Yearbook' 2014 shows that the global electricity demand in 2003 was 14,338 TWh (Terawatt hour) while it increased to 19,878 TWh in 2013. A major portion of this ever increasing 
electricity demand is fulfilled by burning fossil fuels such as coal, oil, natural gas etc. Report shows that approximately $75 \%$ of the electricity comes from these fossil fuels [1]. As the resources are limited, in the near future the world will run out of all fossil fuels. Therefore, the only way to solve the energy crisis is to develop sustainable energy sources. Moreover a large amount of pollutants and toxic gases are emitted from the combustion of fossil fuels which contributes to global warming. The International Energy Agency in 2010 [2] reported approximately $31 \%$ of the total carbon dioxide causing the greenhouse effect which is about 49.5 gigatonnes comes from heat and electricity production fields. Conventional heat engines such as steam turbines are powered by burning fossil fuels and their typical efficiency ranges from 30-40\% [3-6]. Hence, the rest of the heat as well as the byproducts of combustion causes an adverse effect on the environment such as global warming [7-9]. Some reports have identified the negative effects of waste heat including the forming of urban heat islands and global warming $[10,11]$. TEGs are considered as the most promising sustainable energy source to generate electricity as it can utilize the waste heat to transform it into electricity $[12,13]$. These devices harvest electric power from heat energy.

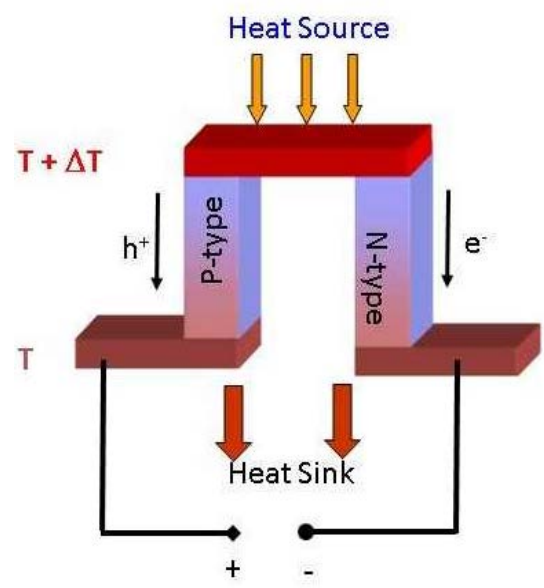

(a)

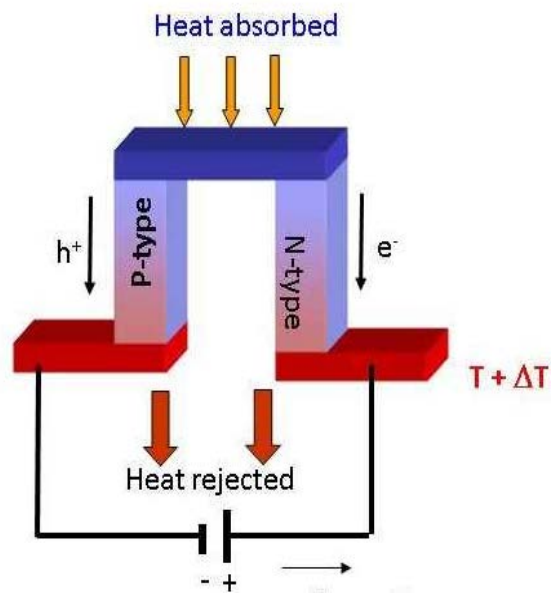

(b)

Figure 1: (a) Energy conversion (Seebeck effect) and (b) refrigeration (Peltier effect) [14].

Figure 1 shows a single unit TEG prepared by combining a p-type and an ntype semiconductor material. An actual TEG contains a series of such junctions of $\mathrm{p}$ and n-type materials. When the temperature gradient is maintained at the junctions of these two dissimilar materials, it produces electricity. The basics behind this electricity generation is when heat is applied to the generator, the negative electrons in the n-type element and the positive holes in the p-type element diffuse from the hot side to the cold side that creates a flow of charge carriers inside the loop or circuit of the device and these flow of charges are 
actually the resulted electricity. This principle is called the Seebeck effect which was discovered in 1821 by Thomas Johann Seebeck. These devices do not have any moving parts creating vibration and sound pollution or exhale any toxic residuals caused by chemical reactions. These eco-friendly devices also possess high scalability and ensure maintenance free long operating life [15-19].

The performance of a TEG is determined by a dimensionless parameter 'Figure of Merit', ZT. ZT depends on three properties of the material. The parameters are: the thermal conductivity $(\kappa)$, electrical conductivity $(\sigma)$ and the Seebeck coefficient (S). If $\mathrm{T}$ is the absolute temperature then the expression for figure of merit is $Z T=\frac{S^{2} \sigma}{\kappa} \mathrm{T}$.

$\mathrm{S}^{2} \sigma$ is sometimes expressed as the term 'power factor'. Power factor gives an idea about the magnitude of thermoelectric power a TEG can generate. It is said that a good TEG should have a power factor in excess of $0.001 \mathrm{~W} / \mathrm{mK}^{2}$. A study reports that a TEG requires a ZT greater than 4 to compete the existing conventional power generation technique [21, 22]. The highest ZT achieved until today ranges from 2.2 to 3.5 at a temperature of 300-915K [21, 22]. However, even if ZT reaches 4, researchers suggest that TEGs are suitable for decentralized smaller scale applications [21]. Among these applications the best one is the engine heat recovery system. Utilizing TEG in automobiles will improve by $10 \%$ in the fuel economy. It is predicted that TEGs will find their maximum applications in wireless sensors, consumer electronics, military and aerospace equipment and waste heat recovery in automobiles [23-25]. Major portions of the research in thermoelectrics were dominated by inorganic materials untill some years back. Due to limited resources however, high cost as well as the complicated synthesis process and environmental risks of inorganic elements, people are getting more interested in organic materials. Organic electronic materials are electronically conducting as they are based on carbon and small molecules. They also intrinsically possess very low thermal conductivity, $(\kappa)$ generally from 0.05 $\mathrm{W} / \mathrm{m} \cdot \mathrm{K}$ to $0.6 \mathrm{~W} / \mathrm{m} \cdot \mathrm{K}[26,27]$. They are becoming a promising alternative in the semiconductor electronics industry because of their availability, ease of mass production and cost effectiveness. They are also environmentally friendly. However, the current maximum value achieved in organic thermoelectrics is 0.43 and due to the chemical stability, the operating temperature window is still very small $(<150)$.

In this paper, a post treatment process of conducting polymer PEDOT: PSS using nitric acid $\left(\mathrm{HNO}_{3}\right)$ and the effect of this treatment on the electrical property of the polymer is reported. The possible reason behind this property enhancement was also mentioned.

\section{PEDOT: PSS}

PEDOT: PSS is a mixed polymer comprising of two ionomers (Figure 2). One element of this mixture is polythiophene based conjugated polymer, poly (3, 4-ethylenedioxythiophene) having a positive charge. The other element is polystyrenesulfonate with the sulfonyl group being deprotonated and carries a 
negative charge. The charged molecules form salt together. PEDOT itself is not soluble in water which means dealing with PEDOT alone was quite difficult. Therefore PSS was added to PEDOT that made the PEDOT: PSS polymer mixture soluble in water. However PSS is insulating in nature therefore it reduces the overall electrical conductivity of the polymer mixture. Alternatively, it is possible to tune up the electrical conductivity through chemical treatment. In addition, it has higher mechanical flexibility, processability, thermal stability and transparency [11]. It can also be a substitute of ITO as transparent electrodes as ITO are depleting [12].

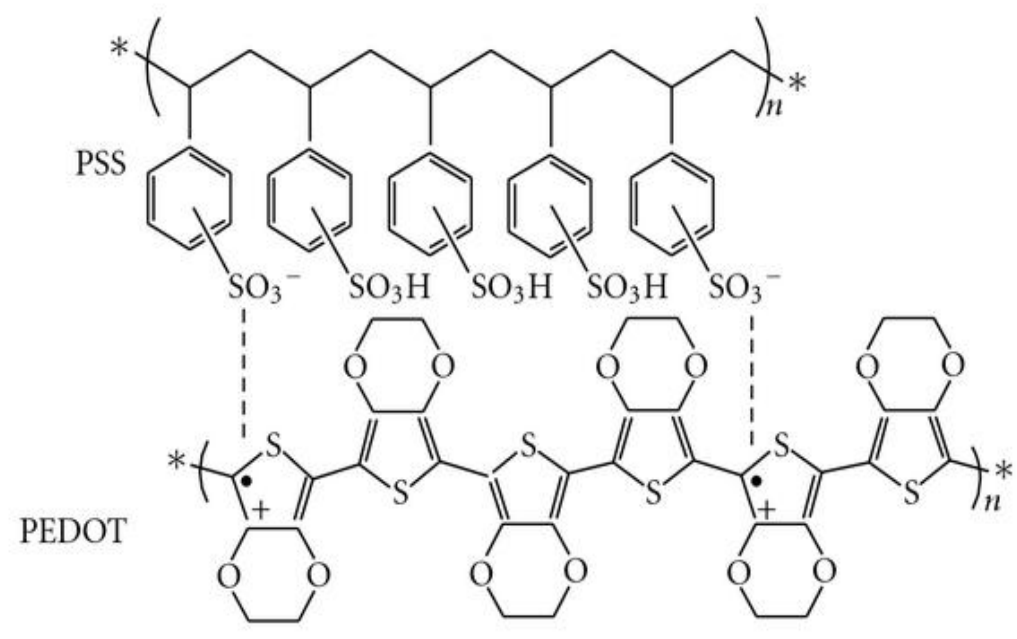

Figure 2: $\quad$ Structure of PEDOT: PSS polymer mixture [28].

\section{Experiment and discussion}

In this experiment, the commercial grade PEDOT: PSS from Sigma Aldrich was used to prepare solid films on glass substrate using the drop casting method. Deionized water was added to the $65 \%(\mathrm{v} / \mathrm{v}) \mathrm{HNO}_{3}$ in different volume ratios to vary the concentration of the acid solution. The samples were then acid treated using nitric acid of different concentrations. After the treatment, the electrical conductivity of the films was measured using the 4-point probe technique. The 4-point probe is the most commonly used technique to measure the sheet resistance of semiconductor samples. Among the 4 probes, a current is passed through the outer two probes while the inner pair of probes sense the voltage generated (Figure 3).

The thickness of the sample is also required to calculate the sheet resistance. A surface profiler was used to determine the sample thickness. The electrical conductivities were calculated by taking the inverse of the resistance values. The results from the electrical conductivity measurement are shown in Figure 4. 


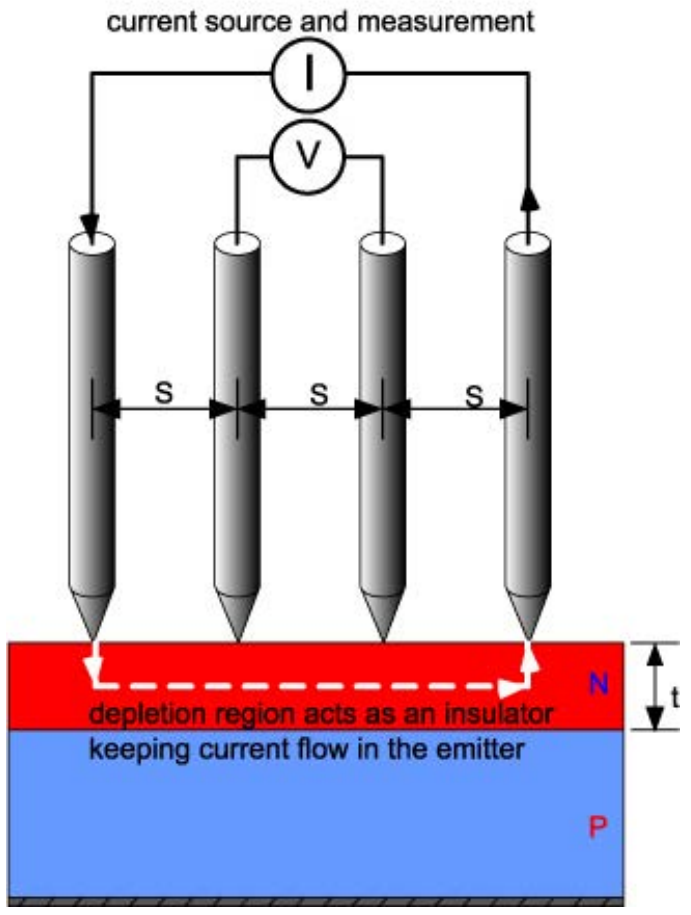

Figure 3: $\quad$ Four-point probe measuring resistivity of a solar cell [29].

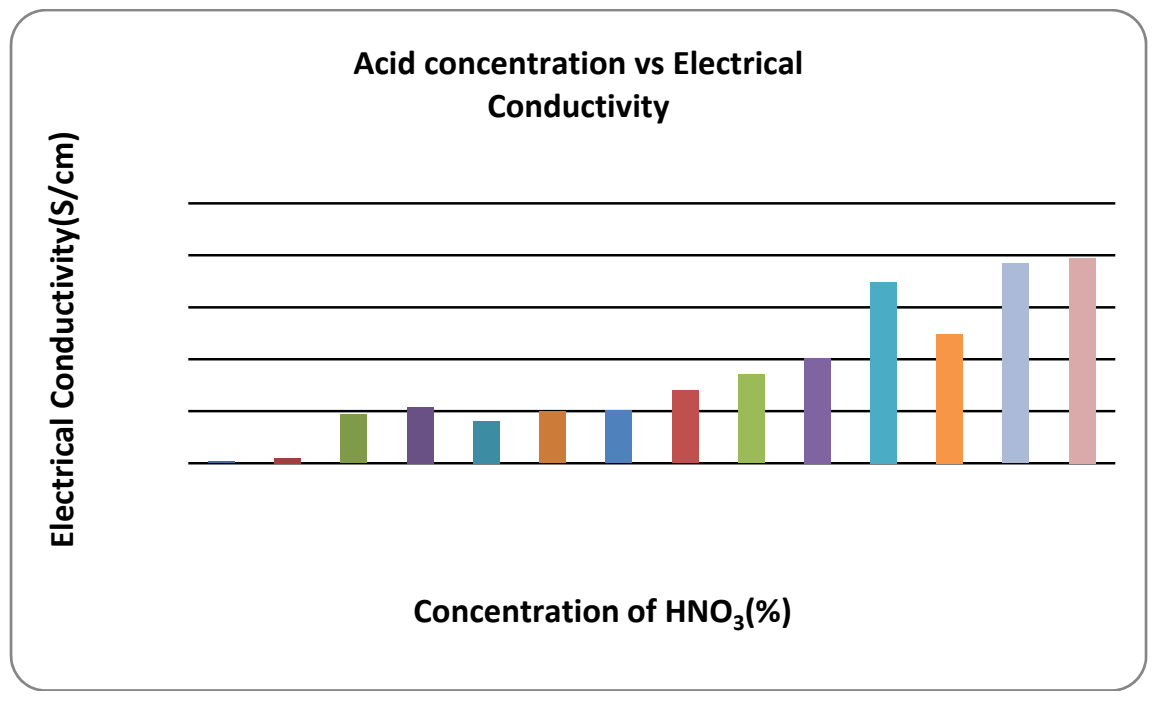

Figure 4: Electrical conductivity of PEDOT: PSS films treated with HNO3 of different concentrations. 
From the figure it can be seen that after the acid treatment, the electrical conductivity of the films increased. Moreover, by increasing the concentration of the acid solution, results improved significantly. The maximum electrical conductivity observed during this experiment was $197.51 \mathrm{~S} / \mathrm{cm}$ when treated with $65 \%$ (v/v) $\mathrm{HNO}_{3}$ while it was only $1.71 \mathrm{~S} / \mathrm{cm}$ for pristine PEDOT: PSS films. Therefore it was an improvement of about 115 times compared to the untreated samples.

To check the compatibility of the prepared films to withstand outdoor conditions, the stability test was performed on films. After around 10 days of observation, it is found that the sheet resistance of the acid treated films appear to be more stable while the resistance of the untreated films increased with the passage of time. This is another advantage over the untreated samples.

Typically, the operating time of a good TEG exceeds 100,000 hours [30, 31]. It is expected that the prepared films will give longevity. Looking for an alternative will not be a problem as organic conducting polymers are available, less expensive and are easy to synthesize or modify if the prepared films are short lived. Furthermore, TEGs are scalable and compact, so they can be modified to meet a range of electricity needs, either domestic or industrial [32, 33].

\section{Conclusion}

In summary, it can be said that this paper provided a simple, easy and cost effective method of improving the thermoelectric property of a polymer. It took less time and effort to achieve wanted results. The as prepared films can be an effective element of a TEG after necessary modification. The films were also quite stable in nature during the observation period. Hence, TEGs will be an important alternative solution in the present time as well as the future for electricity generation. It will also reduce the adverse effects on environment due to the burning of fossil fuels and thus will take us a step closer in the quest to finding sustainable electricity generation.

\section{References}

[1] Energy Outlook 2013: A View to 2040, Exxon Mobil Corporation, 2013.

[2] $\mathrm{CO}_{2}$ emissions from fuel combustion 2012 - Highlights, IEA, 2012.

[3] Yazawa, K. \& Shakouri, A., Cost-Efficiency Trade-off and the Design of Thermoelectric Power Generators. Environ. Sci. Technol., 45, pp. 75487553, 2011.

[4] Ismail, B.I. \& Ahmed, W.H., Thermoelectric Power Generation Using Waste-Heat Energy as an alternative Green Technology. Recent Patents on Electrical Engineering, 2, pp. 27-39, 2009.

[5] Kehlhofer, R., Rukes, B., Hannemann, F. \& Stirnimann, F.X., CombinedCycle Gas and Steam Turbine Power Plants, PennWell, 2009.

[6] Ewing, J.A. The Steam-engines and Other Heat-engines, Cambridge Univ. Press, 2013. 
[7] Bose, B.K., Global Warming: Energy, Environmental Pollution, and the Impact of Power Electronics. Industrial Electronics Magazine, 4, pp. 6-17, 2010.

[8] Castro Villela, I.A.de. \& Silveira, J.L., Ecological efficiency in thermoelectric power plants, - Applied thermal engineering. Appl. Therm. Eng. 27, pp. 840-847, 2007.

[9] Cârdu, M. \& Baica, M., Regarding a new variant methodology to estimate globally the ecologic impact of thermopower plants. Energ. Convers. Manage, 40, pp. 1569-1575, 1999.

[10] Zevenhoven, R. \& Beyene, A. The relative contribution of waste heat from power plants to global warming. Energy, 36, pp. 3754-3762, 2011.

[11] Zhang, G.J., Cai, M. \& Hu, A. Energy consumption and the unexplained winter warming over Northern Asia and North America. Nature Clim. Change, 3, pp. 466-470, 2013.

[12] Hsu, C.T., Huang, G.Y., Chu, H.S., Yu, B. \& Yao, D.J., Experiments and simulations on low-temperature waste heat harvesting system by thermoelectric power generators. Appl. Energy. 88, pp. 1291-1297, 2011.

[13] Hsiao, Y.Y., Chang, W.C. \& Chen, S.L., A mathematic model of thermoelectric module with applications on waste heat recovery from automobile engine. Energy, 35, pp. 1447-1454, 2010.

[14] http://maeweb.ucsd.edu/

[15] Hochbaum, A. I., Chen, R. K., Delgado, R. D., Liang, W. J., Garnett, E. C., Najarian, M., Majumdar, A. \& Yang, P. D., Nature, 451, pp. 163, 2008.

[16] Harman, T. C., Taylor, P. J., Walsh, M. P., \& LaForge, B. E., Science, 297, p. 2229, 2002.

[17] Sales, B. C., Mandrus, D. \& Williams, R. K., Science, 272, pp. 1325, 1996.

[18] Bell, L. E., Science, 321, p. 1457, 2008.

[19] Sootsman, J. R., Chung, D. Y. \& Kanatzidis, M. G., Angew. Chem., Int. Ed., 48, p. 8616, 2009.

[20] Minnich A. J., Dresselhaus, M. S., Ren Z. F., \& Chen G., Energy Environ. Sci., 2, p. 466, 2009.

[21] Vining, C.B. An inconvenient truth about thermoelectric. Nat. Mater. 8, pp. 83-85, 2009.

[22] Anatychuk, L.I. \& Kuz, R.V., Materials for Vehicular Thermoelectric Generators. J. Electron. Mater. 41, pp. 1778-1784, 2012.

[23] Zervos, H., Thermoelectric Generators: A US\$750 million market by 2022. Energy Harvesting Journal, http://www.energyharvestingjournal.com/articles/thermoelectricgenerators-a-750-million-market-by-2022-00004631.asp

[24] Rowe, D.M. United States Thermoelectric Activities in Space, Proc. VIII Intl. Conf. on Thermoelectric Energy Conversion, Nancy, France, pp. 133142, 1989.

[25] Rowe, D.M., Space applications By G. Bennett (Chapter 41) (Ed.), CRC Handbook of Thermoelectrics, CRC Press, 2005.

[26] Crispin X., Jakobsson F. L. E., A. Crispin et al., The origin of the high conductivity of poly(3,4-ethylenedioxythiophene)-poly (styrenesulfonate) 
(PEDOT-PSS) plastic electrodes. Chemistry of Materials, 18(18), pp. 43544360, 2006.

[27] Yan, H. \& Okuzaki, H., Effect of solvent on PEDOT/PSS nanometer-scaled thin films: XPS and STEM/AFM studies. Synthetic Metals, 159(21-22), pp. 2225-2228, 2009.

[28] http://www.hindawi.com/journals/ijp/2009/650509/

[29] http://pveducation.org/pvcdrom/characterisation/four-point-proberesistivity-measurements.

[30] Ismail, B.I. \& Ahmed, W.H., Thermoelectric Power Generation Using Waste-Heat Energy as an alternative Green Technology. Recent Patents on Electrical Engineering, 2, pp. 27-39, 2009.

[31] Aggarwal, R.K. \& Markanda, S., A review on thermocouple for power generation. J. Appl. Sci. Eng. Res. 1, pp. 99-105, 2012.

[32] Synder, G.J., Small Thermoelectric Generators. Electrochem. Soc. Interface, 17, pp. 54-56, 2008.

[33] Zervos, H., Thermoelectric Energy Harvesting 2013-2023: Devices, Applications, Opportunities, IDTechEX Report, 2013. 